\title{
Deutsch nach Englisch (oder parallel) in der Realität einer tschechischen Schule
}

\author{
Alice Brychová
}

At the present time, more attention should be paid to foreign language learning as languages are a means of international communication in today's globalized world. Recent multilingual education policies embrace the idea that several languages should be learnt either simultaneously or sequentially. This article contributes to answering the question, whether and how this goal can be achieved in practical situations at Czech schools.

Multilingualism - German as a second foreign language - German as a Tertiary Language German after English - Foreign Language Teaching

Dem Sprachenlernen sollte in der heutigen Zeit mehr Aufmerksamkeit gewidmet werden, weil die Sprachen ein Mittel der internationalen Kommunikation in einer zunehmend globalisierten Welt sind. Dieses Ziel wird im Rahmen der Mehrsprachigkeitsbildungspolitik um den Gedanken, dass mehrere Fremdsprachen gleichzeitig oder nacheinander gelernt werden sollten, weiterentwickelt. Zur Antwort, ob und wie dieses Ziel in konkreten Situationen im schulischen Unterricht zu realisieren ist, wollte ich mit diesem Artikel einen Beitrag leisten.

Mehrsprachigkeit - Deutsch als zweite Fremdsprache - Tertiärsprachendidaktik - Deutsch nach Englisch - Fremdsprachenunterricht

\section{Besonderheiten des Unterrichtsfaches Deutsch als zweite Fremdsprache}

Das Erlernen einer ersten Fremdsprache garantiert nicht in ausreichendem Maße die Ausbildung einer Kompetenz, die es wirklich ermöglichen würde uns anderen Gemeinschaften und Kulturen anzunähern. Erst das Erlernen einer zweiten Fremdsprache (L3) schafft die Voraussetzung für eine Erziehung zur Mehrsprachigkeit. Unterstützung der Mehrsprachigkeit gehört zu den wichtigen bildungspolitischen Zielen des erweiterten Europas. In diesem Sinne äußern sich auch Dikova/Mavrodieva/Stankulowa (2012), wenn sie darauf hinweisen, dass das Lehren und Lernen einer weiteren Fremdsprache in keinem europäischen Land als eine zusätzliche belastende Verpflichtung verstanden werden sollte, sondern als eine Möglichkeit, die Schüler auf das lebenslange Fremdsprachenlernen vorzubereiten und ihre Lernstrategien und kommunikative Kompetenz zu erweitern. Für die schulische Praxis stellt das gleichzeitige Lernen von mehreren Fremdsprachen jedoch eine Herausforderung dar, und dies besonders dann, wenn dem L3-Unterricht alle traditionellen Bildungsziele des Fremdsprachenunterrichts, die für nur eine Fremdsprache formuliert wurden, aufgebürdet werden und sein spezifischer Stellenwert nicht genügend berücksichtigt wird. Für diesen Fremdsprachenunterricht gilt, dass Schüler einerseits die L3 in einem Alter lernen, in dem ihre kognitiven 
Fertigkeiten schon besser entwickelt sind und sie bereits Erfahrungen mit dem Sprachenlernen haben. Zugleich aber gibt es andere, beschränkende Spezifika dieses Unterrichts, die in Betracht genommen werden müssen und die sich auf die Lernbedingungen im L3-Unterricht (im Vergleich zu denen im L2-Unterricht) negativ auswirken können. Versuchen wir diese Spezifika näher zu betrachten:

1) Negativ beeinflusst das Erlernen einer zweiten Fremdsprache die Tatsache, dass für den L3-Unterricht in der Regel weniger Unterrichtsstunden geplant und dementsprechend auch niedrigere Unterrichtsziele vorgesehen sind. Mit niedrigeren Zielen kann eine niedrige Aktivität und Aufmerksamkeit verbunden sein. Für schwächere Schüler könnte auf der anderen Seite diese Zielsetzung als etwas Erreichbares wahrgenommen werden und deswegen auch motivierend wirken. Ziele des Fremdsprachenunterrichts richten sich in Tschechien heute allgemein nach den Vorgaben, die im sg. Rahmenbildungsprogramm, einem Rahmencurriculum, formuliert sind (vgl. RVP 2007). Die Bestimmung des zu erreichenden Sprachniveaus orientiert sich dabei an den Beschreibungen des Gemeinsamen Europäischen Referenzrahmens (vgl. GER online). Für den L3-Unterricht gelten Unterrichtsziele, die dem Sprachniveau A1 entsprechen, die aber alle Fertigkeiten und Sprachaspekte in gleichem Maße wie beim L2-Unterricht betreffen. Diese Ziele scheinen aber angesichts der geringen Stundenzahl und des (anzunehmenden) kleineren Interesses der Lerner an der L3 nur schwierig realisierbar zu sein.

Positiv könnte in diesem Zusammenhang wirken, wenn Deutsch stärker als Sprache unserer Nachbarn präsentiert würde und deutlicher auf den Umstand hingewiesen würde, dass man Österreich oder Deutschland relativ einfach besuchen kann. Beim ersten flüchtigen Kontakt braucht man verstärkt rezeptive Fertigkeiten, wenn man sprechende Muttersprachler, geschriebene Informationen oder andere Texte verstehen möchte. Im Unterricht könnten aus diesem Grund mehr die rezeptiven Fertigkeiten in den Vordergrund gestellt werden. Die Lernenden könnten auch intensiver mit der rezeptiven Kenntnis des Wortschatzes arbeiten und versuchen, auch authentische Texte mit Hilfe von Verstehensstrategien zu verstehen.

2) Das Erlernen der deutschen Sprache als L3 beginnt in einem anderen Alter als das Erlernen der ersten Fremdsprache Englisch. Englisch lernen schon kleine Kinder im Kindergarten oder auf der Primarstufe. Der spätere Beginn des Unterrichts in Deutsch als Fremdsprache (weiter DaF) beeinflusst den Lernprozess sowohl im negativen als auch im positiven Sinne. Negativ kann den L3-Unterricht bei spätem Beginn die Tatsache beeinflussen, dass sich Schüler in der Pubertät weniger aktiv am Unterricht beteiligen und es schwieriger ist, sie zu motivieren. Dieser Tatsache trägt z. B. die Konzeption des Lehrwerks Ideen von Herbert Puchta und Wilfried Krenn (Krenn/Buchta 2010) Rechnung, die anmerken: „Jugendliche zu unterrichten ist eine interessante, aber nicht immer leichte Aufgabe. Die Ursachen für viele 
Probleme liegen oft in der komplexen entwicklungsbedingten Situation, in der sich die Jugendlichen in diesem Alter befinden. In der Übergangsphase von der Kindheit zum Erwachsenenleben müssen sie ihre eigene Identität neu definieren."

Positiv könnte aber auf das Lernen im L3-Unterricht allgemein wirken, dass die Interessensbereiche von älteren Schülern im Vergleich zu jüngeren Schülern viel weiter sind und somit im Unterricht auch inhaltlich anspruchsvollere Themen behandelt werden können. (Sprachlich sind die Schüler jedoch noch eingeschränkt, und dies führt zu Hemmungen beim Versprachlichen ihrer Gedanken, was wiederum eine negative Wahrnehmung der Situation verursachen kann.) Sie haben außerdem schon Erfahrungen mit dem Lernen einer Fremdsprache, die sie zu ihren Gunsten nutzen können, so dass sie beim L3-Lernen schneller vorankommen. Gerade bei Deutsch nach Englisch könnte den Lernern durch geeignete Aufgaben und Übungen die Verwandtschaft beider Sprachen bewusst gemacht werden, weil Schüler in diesem Alter schon fähig sind, über Sprachen zu reflektieren. Diese Suche nach Parallelen und Unterschieden kann aber zeitlich ziemlich anspruchsvoll sein. Außerdem ist die Voraussetzung dafür, dass auch die Lehrer/-innen beide Sprachen beherrschen, was eigentlich eher selten der Fall ist, weil die Mehrsprachigkeit in der Ausbildung erst vor kurzem zum Thema geworden ist. Lehrer/-innen wurden bisher als Experten/-innen für nur eine Fremdsprache ausgebildet, die sie dann auf einem möglichst hohen Niveau beherrschen sollten.

3) Unter Fremdsprachenlehrern besteht die allgemein verbreitete Erwartung, dass die Motivation zum Erlernen der L3 anders, und zwar gewöhnlich niedriger als bei der L2 ist. Im Falle, dass die L2 Englisch - also die weltweite lingua franca - ist, kann man vermuten, dass das Interesse, eine weitere Fremdsprache zu lernen, niedrig ist. In diesem Zusammenhang stellt sich die Frage, wie motivierend kommunikativ formulierte Lernziele für L3-Lerner sein können, die für eine internationale Kommunikation genauso gut ihre erste Fremdsprache Englisch benutzen können. In Tschechien bleibt für die Wahl der deutschen Sprache als L3 in jedem Fall das Argument, dass man mit Hilfe von Deutsch in den Nachbarstaaten zurechtkommen kann. Eine selbst erlebte Situation im deutschsprachigen Ausland, bei der der betreffende Schüler erfolgreich sprachlich auf die deutschsprachige Realität reagiert, könnte ihm bzw. ihr eine positive Perspektive für die Anwendung von Deutschkenntnissen aufzeigen.

4) Deutsch als zweite Fremdsprache wird nur als Wahlfach unterrichtet und muss deswegen mit Fächern wie Sport oder Computerunterricht konkurrieren. Viele DaF-Lehrer/-innen klagen über eine sinkende Zahl von Deutschlernenden. Diese Tendenz kann man wohl auf die oben angeführten Gründe zurückführen. Außer einer niedrigen Zahl an Schülern kommen als weiterer negativer Faktor noch die oftmals ungünstigen Unterrichtszeiten hinzu: Oft findet der Wahlunterricht 
entweder am frühen Morgen oder in den späten Nachmittagsstunden statt, also zu Zeiten, zu denen sich Schüler nicht wirklich gut konzentrieren können. Umso mehr sollten DaF-Lehrer/-innen methodisch-didaktisch ausgestattet sein, um den Unterricht effizient zu organisieren und mit den Schülern die erwünschten (oder gar vorgeschriebenen) Unterrichtsziele zu erreichen.

\section{Tertiärsprachendidaktik und ihre Umsetzung in der schulischen Praxis}

Für das Lernen des Deutschen als Fremdsprache nach oder parallel zu Englisch (DaFnE) entsteht eine besondere Situation, für die nach Neuner (2003: 7-10) einige didaktische Prinzipien gelten, die sich im Rahmen der inzwischen etablierten Tertiärsprachendidaktik entwickelt haben. Es handelt sich dabei um Prinzipien, die das L3-Lernen unterstützen und Prozesse beim L2- und L3-Lernen berücksichtigen. Beim Prozess des Fremdsprachenlernens sind zwei Hauptaspekte wichtig: das Sprachbewusstsein und das Sprachlernbewusstsein. Im ersten Fall handelt es sich um das Wissen und Können in bereits gelernten Sprachen, im zweiten Fall um die Erfahrungen, die beim Fremdsprachenlernen oft individuell und unbewusst gemacht wurden und die weiterentwickelt werden sollten.

Bei den genannten Prinzipien der Tertiärsprachendidaktik handelt es sich um das kognitive Lernen und Lehren, das den Vergleich beider Sprachen in allen ihren Teilaspekten und eine Diskussion über die Unterschiede und Gemeinsamkeiten ermöglicht. Weiter wirken auf das L3-Lernen unterstützend Prinzipien wie Verstehen als Grundlage des Lernens und Textorientierung. Diese Prinzipien gehen davon aus, dass im Unterricht mit Texten gearbeitet werden sollte, die es den Lernenden ermöglichen, von Anfang an relativ viel aufgrund ihrer Englisch-, Tschechischund Weltkenntnisse zu verstehen. Die Rezeption der verwendeten Texte kann auch durch die Wahl bekannter Themen unterstützt werden, was eine Kombination mit dem nächsten Prinzip darstellt, nämlich mit der Inhaltsorientierung. Mit diesem Prinzip wird auch angedeutet, dass Inhalte und Themen für das Lernen sehr wichtig sind. Wenn die genannten Prinzipien im Deutschunterricht zur Geltung kommen, ist zu vermuten, dass Lernende im Unterricht die neue Sprache schneller verstehen und später auch relativ erfolgreich produzieren. Als Synergieeffekt aus den vorher genannten Prinzipien ergibt sich das letzte Prinzip, das als Ökonomisierung des Lernprozesses bezeichnet wird.

Es bleibt aber offen, inwieweit Lehrende diese Prinzipien überhaupt kennen, ob Sie wissen, wie man eine Tertiärsprache unterrichten kann. Aus der Praxis sind eher Stimmen zu hören, die Englischkenntnisse bei Deutschlernern als problematisch empfinden, weil sich diese in Bezug auf die Deutschkenntnisse als Störfaktor bemerkbar machen. Ebenfalls sollte untersuchtwerden, welche Erfahrungen Lernende bei ihrem L2-Unterricht gemacht haben und ob sie sich dessen bewusst sind, dass sie ihre L1- und L2-Lernerfahrungen auch im L3-Unterricht weiterentwickeln können. 
Vielleicht handelt es sich bei der Forderung nach Erfahrungstransfer von L2 nach L3 für Schüler um eine Devise, mit der sie nichts anfangen können. Bei einem gewissen Grad der Steuerung seitens der Lehrer/-innen oder Lehrwerke könnten Lernende an ihre bereits erworbenen Sprachkenntnisse und Lernerfahrungen anknüpfen. $\mathrm{Ob}$ diese Möglichkeit der Ökonomisierung des L3-Lernens heute bereits tatsächlich auch im realen Unterricht ausgenützt wird, was Schüler über das gleichzeitige Lernen von zwei Fremdsprachen in der Schule denken und ob sie gewisse Parallelen beim Lernen von beiden Fremdsprachen empfinden, wollte ich an einer tschechischen Schule in Brünn feststellen.

\section{Eine empirische Untersuchung zur Einstellung der Schüler zu ihrem Fremdsprachenunterricht}

\subsection{Fragestellungen und Untersuchungsobjekt}

Aus den oben genannten Gründen habe ich im Frühjahr 2012 eine erste Probeuntersuchung an einer Grundschule (1. bis 9. Jahrgangsstufe) vorgenommen. Auf der Primar- und Sekundarstufe I dieser Schule lernen insgesamt 800 Schüler. Es handelt sich um eine typische Schule in einem Neubauviertel der Stadt Brünn, die sich in erster Linie nicht auf das Fremdsprachenlernen spezialisiert, sondern auf den Sportunterricht. Diese Schule organisiert aber regelmäßig Exkursionen für Schüler/innen in die deutschsprachigen Länder und unterhält eine Schulpartnerschaft mit einem Sportgymnasium in Stuttgart. ${ }^{1}$

Die Hauptfragen, die am Anfang meiner Untersuchung standen, waren:

1. Was denken Schüler/-innen von dem parallelen Lernen von zwei Fremdsprachen?

2. Sind sie sich ihrer auf diese Art erworbenen Mehrsprachigkeit bewusst?

3. Mit welchen Zielen verbinden Schüler/-innen ihren Fremdsprachenunterricht in Englisch und Deutsch?

4. Können sie gewisse Parallelen im Englisch- und Deutschunterricht selbst erkennenund dies auch für die Ökonomisierung ihres Tertiärsprachenlernens nutzen?

Meine Thesewar, dass Schüler/-innen dieenglischeSpracheals Kommunikationsmittel für ihre Verständigung im Ausland wahrnehmen und Deutsch nicht besonders gern lernen, weil diese Sprache für sie schwieriger als Englisch und für die Verständigung weniger brauchbar ist. Ich habe weiter vermutet, dass sie deswegen lieber Englisch lernen und diese Sprache auch einfacher finden. Ich habe aber keine reale Vorstellung gehabt, ob die Probanden auch ihre Lernerfahrungen spontan beim Lernen einer dritten Sprache benutzen und was sie davon halten oder ob sie fähig

1 Der Name der Schule wird aus Gründen der Anonymität nicht genannt. 
sind, beim Unterricht über die Gemeinsamkeiten und Unterschieden der beiden Sprachen zu reflektieren. Das letzte Ziel meiner Untersuchung war festzustellen, welche Gemeinsamkeiten die Lernenden in der Methodik des Englisch- und des Deutschunterrichts selbst erkennen.

\subsection{Datenerhebung und -auswertung}

Für die Untersuchung habe ich zwei Instrumente gewählt: Erstens habe ich einen kurzen Fragebogen zusammengestellt, zweitens habe ich die Daten mit den Aussagen einer Deutschlehrerin konfrontiert, mit der ich im Anschluss an die Fragebogenerhebung ein Gespräch geführt habe. Die Fragebögen wurden an 23 Schüler/-innen der 7. Klasse, an 30 Schüler/-innen der 8. Klasse und an 25 Schüler/-innen der 9. Klasse, d. h. an insgesamt 78 Schüler/-innen verteilt. Alle Probanden haben anonym auf Tschechisch formulierte Fragen beantwortet, indem sie aus mehreren Möglichkeiten die zutreffende ausgewählt und markiert haben oder ihre eigene Meinung als Ergänzung ihrer Antwort frei formuliert haben.

\subsubsection{Feststellung der Meinung der Deutschlerner zum Lernen mehrerer Fremdsprachen}

Die meisten Antworten auf die Frage, ob die Schüler glauben, dass es sinnvoll ist, mehrere Fremdsprachen zu lernen, haben bestätigt, dass viele Schüler/-innen sich dessen bewusst sind, dass sie ihre Möglichkeiten zur Verständigung im Ausland dadurch erweitern, mögliche authentische Kommunikationssituation situieren sie aber in die Zukunft und schreiben, dass wenn sie einmal Erwachsen sein werden, sie sich besser im Ausland verständigen können. Andere halten die Kenntnis von mehreren Fremdsprachen für ihre berufliche Karriere für wichtig. Eine gewisse Zahl von Probanden (7) hat diese Frage überhaupt nicht beantwortet. Die Gründe können unterschiedlich sein, aber es gibt auch (9) eindeutig ablehnende Antworten auf diese Frage. Entweder wurde schlichtweg behauptet, dass es nicht gut ist, mehrere Fremdsprachen gleichzeitig zu lernen, oder es wurde noch der Grund für diese Meinung genannt: Man verwechselt beim parallelen Lernen der beiden Sprachen Vieles, man ist verwirrt. Ein Schüler hat sogar mehrmals wiederholt, dass es überflüssig ist Deutsch zu lernen und dass Englisch eine nützlichere Sprache ist und zum Schluss bemerkte er explizit: „Ich will Deutsch nicht lernen.“ Ein anderer Schüler argumentiert für Deutsch, weil man nicht weit fahren muss, um die Sprache benutzen zu können. Den Wunsch, nur eine Fremdsprache zu lernen, unterstützen die Meinungen, dass es besser wäre eine Fremdsprache intensiver zu lernen und wirklich gut zu beherrschen, bevor man eventuell beginnt, eine zweite Fremdsprache zu lernen. Nur eine Schülerin unterstreicht die Wichtigkeit der Mehrsprachigkeit und empfiehlt sogar die Möglichkeit eine dritte Fremdsprache zu lernen. Es lässt

Brünner Hefte zu Deutsch als Fremdsprache • Jahrgang 5 • Nummer $1 \bullet 2012$ 
sich sicher vermuten, dass sich die Meinungen der schwächeren Schüler von denen der besseren Schüler unterscheiden, was eine ,aufrichtige“ Antwort bestätigt: „Es ist gut mehrere Sprachen zu lernen, weil ich sprachenbegabt bin“.

\subsubsection{Gründe für das Fremdsprachenlernen und Einstellung zum Fremdsprachenlernen}

Bei den Fragen Nummer 2 und 3 wurden verschiedene Antwortmöglichkeiten angeboten, die über unterschiedliche Aussagen über die Beziehung zur englischen und deutschen Sprache als Sprachen und auch als Unterrichtsgegenständen machen. Die Möglichkeit, dass das Lernen der englischen Sprache den Schülern Spaß macht, haben insgesamt $23(30 \%)$ der Probanden ausgewählt, wobei diese Meinung mit dem Alter steigt. Eine wesentlich größere Gruppe, 53 Schüler (oder 55,5 \%), lernen Englisch, weil die Kenntnis dieser Sprache für die Verständigung in der Welt unabdingbar ist. Das meinen Schüler in allen Klassen gleichmäßig verteilt.

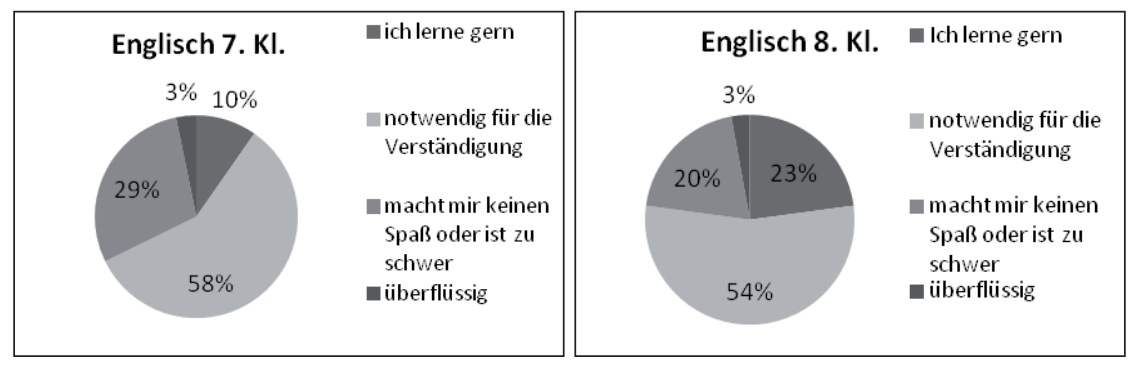

Englisch 9. KI.

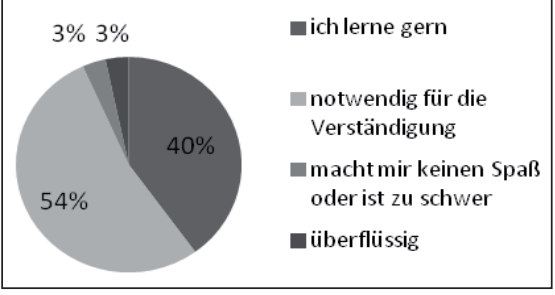

Das Lernen der deutschen Sprache empfinden insgesamt wiederum 22 Probanden (30 \%) als amüsant und sie lernen Deutsch gern. Mit dem Ziel, sich in Nachbarländern verständigen zu können, lernen 37 (fast 50 \%) Schüler Deutsch. 24 Schüler $(30 \%)$ halten Deutsch für eine schwierige Sprache oder das Lernen macht ihnen nicht viel Spaß und 10 Schüler (fast $13 \%$ ) meinen sogar, dass es überflüssig ist, Deutsch zu lernen. Diese Meinung weist eine wachsende Tendenz in der steigenden Jahrgangsstufe auf. Dies könnte dadurch verursacht sein, dass die 
entsprechenden Schüler enttäuscht sind, dass sie in der deutschen Sprache nicht genug gelernt haben, um kommunizieren zu können.
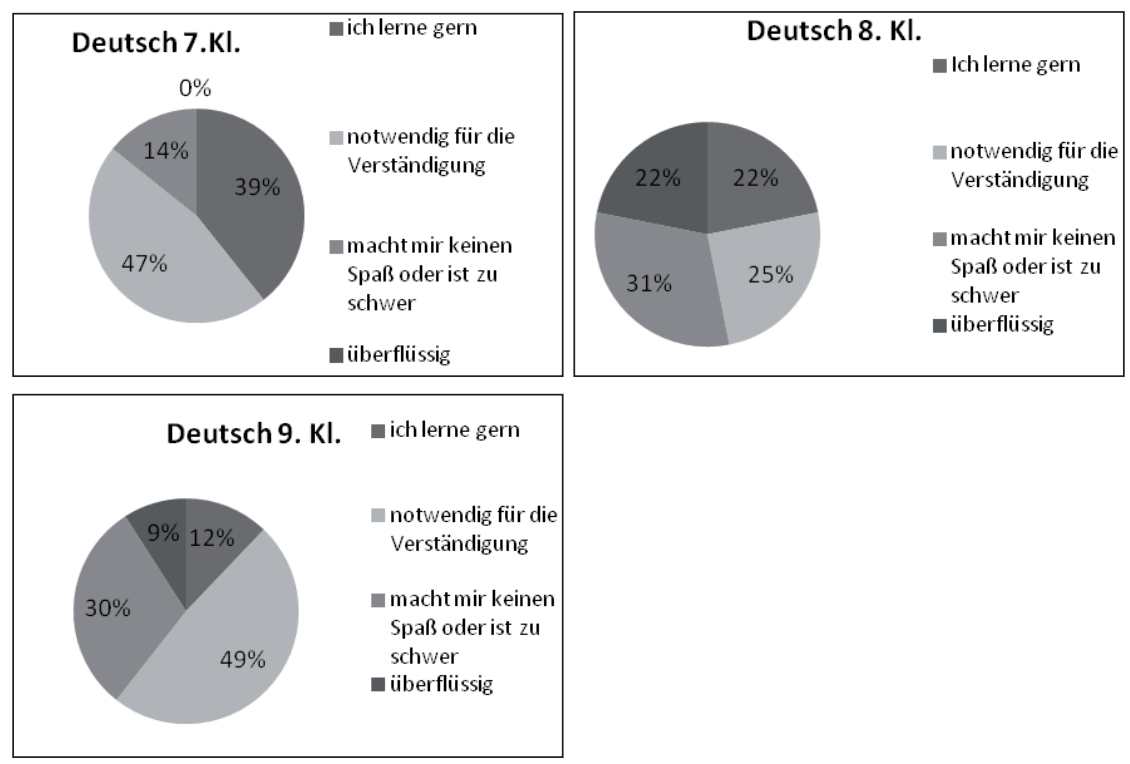

Bei Fragen 2 und 3 wollte ich auch feststellen, wie schwer die Schüler das Lernen von beiden Sprachen empfinden und ob es ihre Einstellung gegenüber den Sprachen beeinflusst. Englisch ist für 17 Lernende (fast $22 \%$ ) eine schwierige Sprache und/ oder macht ihnen nicht besonders Spaß. Diese Aussagen waren stark durch die Beziehung zur jeweiligen Lehrerin beeinflusst und die Meinung über diese Lehrerin wurde auch offen ausgedrückt. In den Fällen, in denen die Deutschlehrerin positiv hervorgehoben wurde und dadurch oft auch der DaF-Unterricht als positiv eingeschätzt wurde, wurde mehrmals angedeutet, dass die Englischlehrerin nicht beliebt ist: „Englisch müsste jemand unterrichten, der es kann.“

\subsubsection{Lernerfahrungen und Sprachwissen aus dem L2-Lernen bewusst anwenden}

In der Frage Nr. 4 wollte ich überprüfen, ob Schüler/-innen ihre Lernerfahrungen, die sie beim Lernen ihrer ersten Fremdsprache gesammelt haben, beim Deutschlernen bewusst anwenden können. Nur 6 Schüler aus der 7 . Klasse, 7 aus der 8. Klasse und 3 aus der 9. Klasse, d. h. insgesamt 16 Schüler (nicht einmal $20 \%$ ), profitieren nach ihrer eigenen Meinung von den Lernerfahrungen und Kenntnissen aus dem Englischunterricht beim Deutschlernen. Insgesamt 9 Schüler 
möchten auf die Erfahrungen zurückgreifen, wissen aber nicht wie, und 39 Schüler, also $50 \%$ Prozent der Probanden, glauben sogar, dass es nicht sinnvoll ist, auf die Lernwege und Erfahrungen beim Englischlernen auch beim Deutschlernen zurückzugreifen. Das Prinzip der Ökonomisierung ist daher meiner Meinung nach in der heutigen Unterrichtspraxis, wie sie die Schüler wahrnehmen, fast nicht zur Geltung zu bringen. Die meisten Schüler/-innen nutzen in den meisten Fällen weder ihre Sprachkenntnisse noch ihre Lernerfahrungen und glauben auch nicht, dass dies sinnvoll wäre. Seitens der Lehrerin gibt es auch wenig Anregungen und Möglichkeiten (im Durchschnitt 17 \%) dazu.
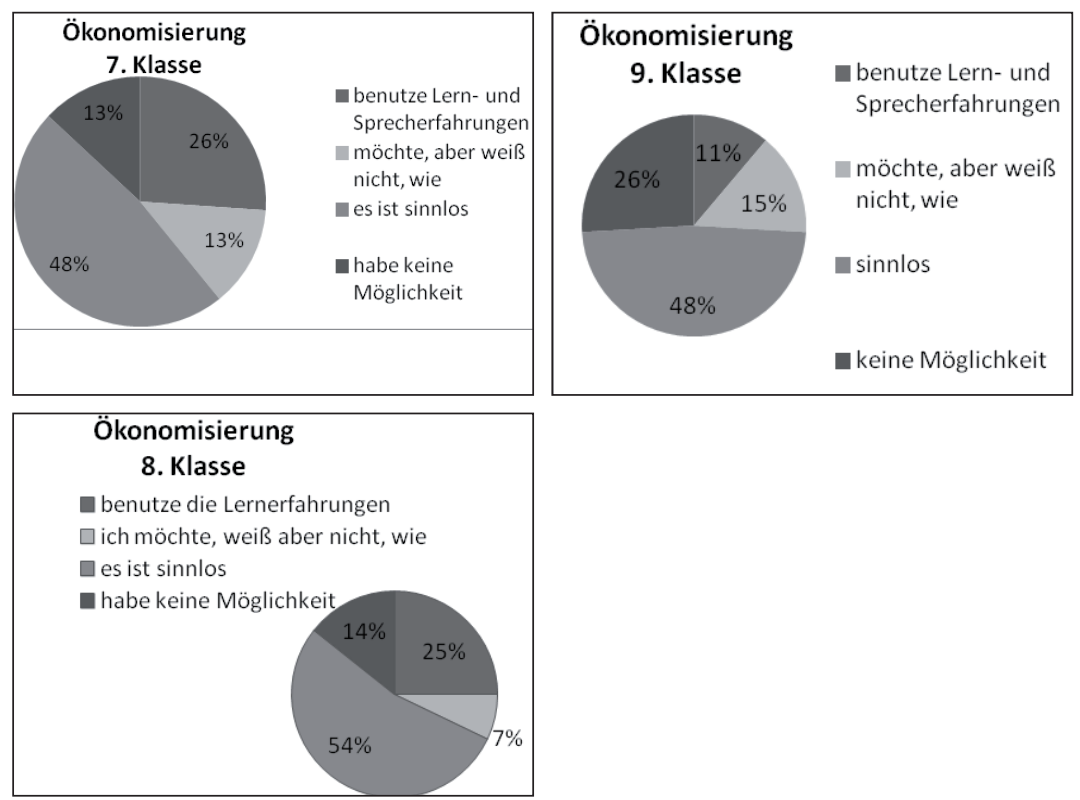

\subsection{4. Ähnlichkeiten beim Lernen beider Fremdsprachen entdecken}

Die Gemeinsamkeiten von Unterrichtskonzeptionen beider Fremdsprachen, wie sie die Schüler/-innen sehen, wollte ich mit der 5. Frage überprüfen. Die Ergebnisse zeigen, dass die meisten Schüler wenige Ähnlichkeiten in beiden Unterrichtsverfahren sehen. Die am häufigsten markierten Komponenten im Unterricht, die nach Meinung der Schüler ähnlich verlaufen, sind die Wortschatzarbeit (33 Schüler) und die Gespräche. Ein Proband hat die fehlende Ähnlichkeit von beiden Sprachunterrichtsverfahren direkt mit folgendem Argument kommentiert: „Im Englischen können wir doch schon mehr, also deswegen kann man nicht mit ähnlichen Texten arbeiten.“ Ein anderer Kommentar lautete: „Den Englisch- und 
Deutschunterricht kann man nicht vergleichen, es gibt keine Ähnlichkeiten, weil die Lehrerinnen anders sind. "Ein weiterer Schüler merkte an: „[...] die Unterschiede sehe ich eben darin, dass es sich um Englisch und Deutsch handelt [...]".
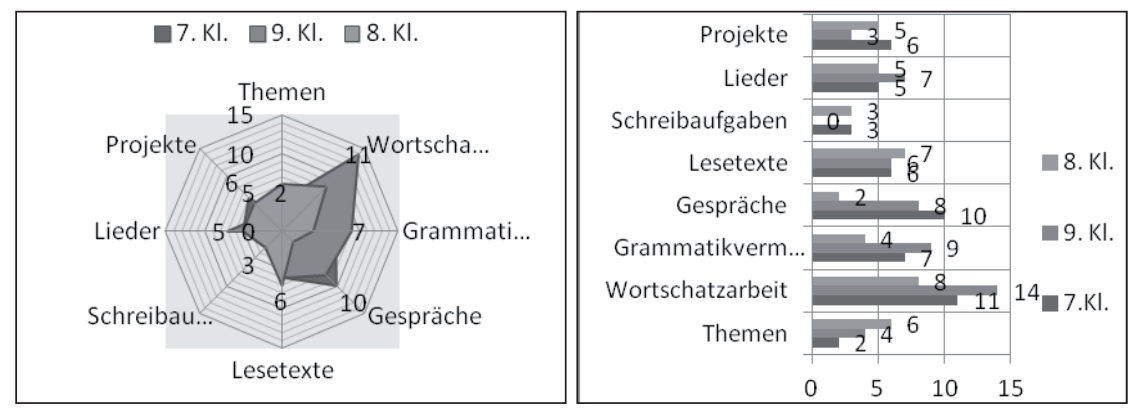

Wiediebeiden Graphikenzeigen, wurdeninder8. Klassein beidenUnterrichtsverfahren am meisten Gemeinsamkeiten bei der Wortschatzarbeit, gefolgt von der Arbeit mit Lesetexten gefunden. Auch in der 9. Klasse empfinden die Schüler sehr deutlich, dass die meisten Gemeinsamkeiten im Fremdsprachenunterricht in der Wortschatzarbeit zu suchen sind, weniger deutlich dann auch bei der Grammatikvermittlung und bei der Arbeit mit Gesprächen. Diese Meinung vertreten auch die Schüler der 7. Klasse am häufigsten.

\section{Zusammenfassung und Perspektiven der Tertiärsprachendidaktik in Tschechien}

Wenn wir die Ergebnisse der eben vorgestellten Pilotuntersuchung aus der Perspektive der Empfehlung der Europäischen Union, außer der Muttersprache zwei weitere Sprachen auf unterschiedlichem kommunikativen Niveau zu beherrschen, vergleichen, sehen wir, dass dieses Ziel während des Schulbesuches an tschechischen Grundschulen (d. h. in den Klassen 1 bis 9) nicht immer einfach zu erfüllen ist.

Der Unterricht in der ersten Fremdsprache ist obligatorisch. Anschließend besuchen einige Schüler überhaupt keinen weiteren Fremdsprachenunterricht mehr. Die meisten Schüler in Tschechien lernen als erste Fremdsprache Englisch und sind zum Lernen einer zweiten Fremdsprache nicht immer stark motiviert. In dieser Hinsicht stellt die Schule, die ich für meine Untersuchung ausgewählt habe, schon fast eine Ausnahme dar. An dieser Schule wählen immer noch alle Schüler eine zweite Fremdsprache. An anderen Schulen in Tschechien wählen nicht alle Schüler eine zweite Fremdsprache, sondern oft ein anderes Wahlfach. Nach dem Bericht der Tschechischen Schulinspektion (ČŠI 2010:6) wählen in der Sekundarstufe 
(Klassenstufe 6 bis 9) im Durchschnitt nur noch $11 \%$ aller Schüler/-innen eine zweite Fremdsprache und die Zahl sinkt immer noch weiter.

Es ist nicht einfach, parallel zwei Sprachen zu lernen, aber bei Deutsch und Englisch als verwandten Sprachen gibt es Möglichkeiten, auf Lernerfahrungen zurückzugreifen und zugleich die Ähnlichkeiten und Unterschiede in den jeweiligen Sprachen zu vergleichen. Damit würden die oben erwähnten Prinzipien des kognitiven Lernens und Lehrens und der Ökonomisierung in der Schulpraxis umgesetzt und die Mehrsprachigkeit der Schüler/-innen entwickelt.

Eine meiner Absichten war zu prüfen, in wie weit Schüler/-innen selbst in der Lage sind, auf ihre L2- Lernerfahrung zurückzugreifen und auf diese Weise ihre Lernprozesse selbständig ökonomisch zu steuern. Deswegen wollte ich auch prüfen, ob Schüler selbst ihre Lernerfahrungen im L3-Unterricht reflektieren, ob sie Parallelen finden. Die Ergebnisse zeigen, dass nicht alle Schüler dazu in der Lage sind, die Prozesse der Ökonomisierung ihres Lernens selbständig zu starten. Die meisten Ähnlichkeiten sehen die Schüler/-innen in der Wortschatzarbeit. Vielleicht meinten auch einige der Respondent/-innen unter Ähnlichkeiten auch den gemeinsamen Wortschatz. Damit diese und andere Gemeinsamkeiten beim Lernen wirklich positiv wirken, müssten die Fremdsprachenlehrer-/innen den Schüler/-innen helfen (das Prinzip des kognitiven Lehrens). Deswegen ist es wichtig, dass auch Fremdsprachenlehrer/-innen Kenntnisse über Tertiärsprachendidaktik haben und minimale Sprachkenntnisse in der anderen Fremdsprache, die ihre Schüler lernen. Die Ergebnisse dieser Stichprobe zeigen, dass wir dieser Problematik mehr Aufmerksamkeit widmen sollten und auch die praktische Umsetzung der sprachenpolitischen Bildungsziele überlegen müssen.

\section{Literaturverzeichnis}

Dikova, Venzislava / Mavrodieva, Lyubov / Stankulowa, Krystyna (2012): Curriculum für Deutsch als Fremdsprache in der bulgarischen allgemeinbildenden Oberschulen. http://zif.spz.tu-darmstadt.de/jg-05-3/beitrag/dikova.htm. (20. 4. 2012).

GER (Gemeinsamer europäischer Referenzrahmen für Sprachen: lernen, lehren, beurteilen) (2011): http://www.goethe.de/z/50/commeuro/deindex.htm. (27. 10. 2011).

Neuner, Gerhardt / Berger, Maria Cristina / Curci, Anna Maria / Gasparro, Antonia (2003): Deutsch nach Englisch: good + gut $=$ ottimo. Mailand, Goethe Institut.

Janíková, Věra / Sorger, Brigitte (2011): Mehrsprachigkeit in der Tschechischen Republik am Beispiel Deutsch nach Englisch. Brno, Tribun EU.

ČŠI (Česká školní inspekce) (2010): Tematická zpráva: Souhrnné poznatky o podpoře a rozvoji výuky cizich jazykì v predškolním, základním a středním vzdělávání v období let 2006-2009. Praha, ČŠI. http://www.csisr.cz/cz/85027-podpora-a-rozvoj-vyukycizich-jazyku. (13. 7. 2010).

Krenn, Wilfried / Puchta, Herbert (2010): Ideen. München, Hueber Verlag.

RVP (2007): http://www.vuppraha.cz/wp-content/uploads/2009/12/RVPZV_2007-071. pdf. (30. 4. 2012). 